\title{
Erratum to: Thin film based semi-active resonant marker design for low profile interventional cardiovascular MRI devices
}

\author{
Engin Baysoy ${ }^{1,3}$ (1) - Dursun Korel Yildirim ${ }^{1} \cdot$ Cagla Ozsoy $^{1} \cdot$ Senol Mutlu $^{2} \cdot$ \\ Ozgur Kocaturk ${ }^{1}$
}

Published online: 12 October 2016

(C) ESMRMB 2016

\section{Erratum to: Magn Reson Mater Phy DOI 10.1007/s10334-016-0586-8}

Unfortunately, the captions of Figs. 5, 6, and 7 were transposed in the original online publication of this article.

The correct figures and captions are given here (Figs. 5, $6,7)$, and the article itself has been corrected.

Fig. 5 Computer aided design drawings of the fabricated RF marker (not to scale). a Cross sectional view and magnified views of RF marker showing thickness of thin film layers deposited on cylindrical catheter tube (PEBAX). b Overall view of the RF resonant marker including all components

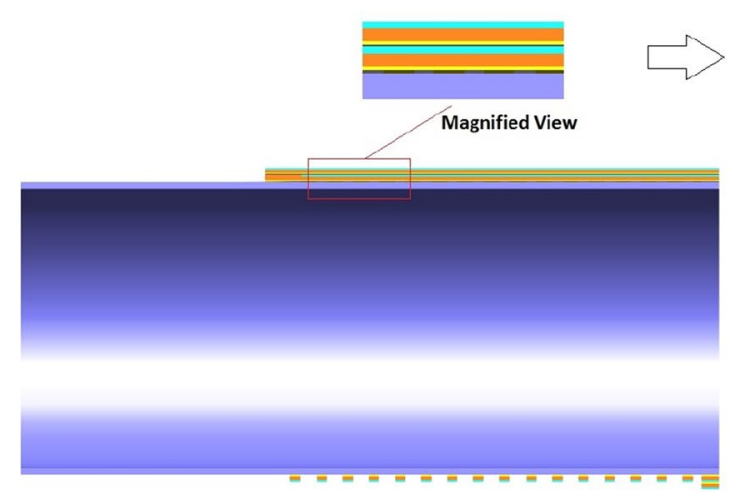

.2 .5 um Parlene Coating $.30 \mu \mathrm{m}$ Cu Electroplating $.100 \mathrm{~nm} \mathrm{Au}$ $.10 \mathrm{nmCr}$

.PEBAX Tube

a

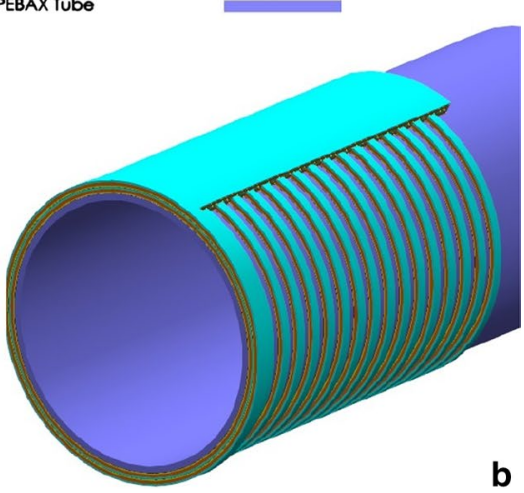

The online version of the original article can be found under doi:10.1007/s10334-016-0586-8.

Engin Baysoy

enginbaysoy@gmail.com

1 Institute of Biomedical Engineering, Bogazici University, Istanbul, Turkey

2 Department of Electrical and Electronics Engineering, Bogazici University, Istanbul, Turkey

3 Vocational School of Biomedical Device Technology, Acibadem University, Istanbul, Turkey 


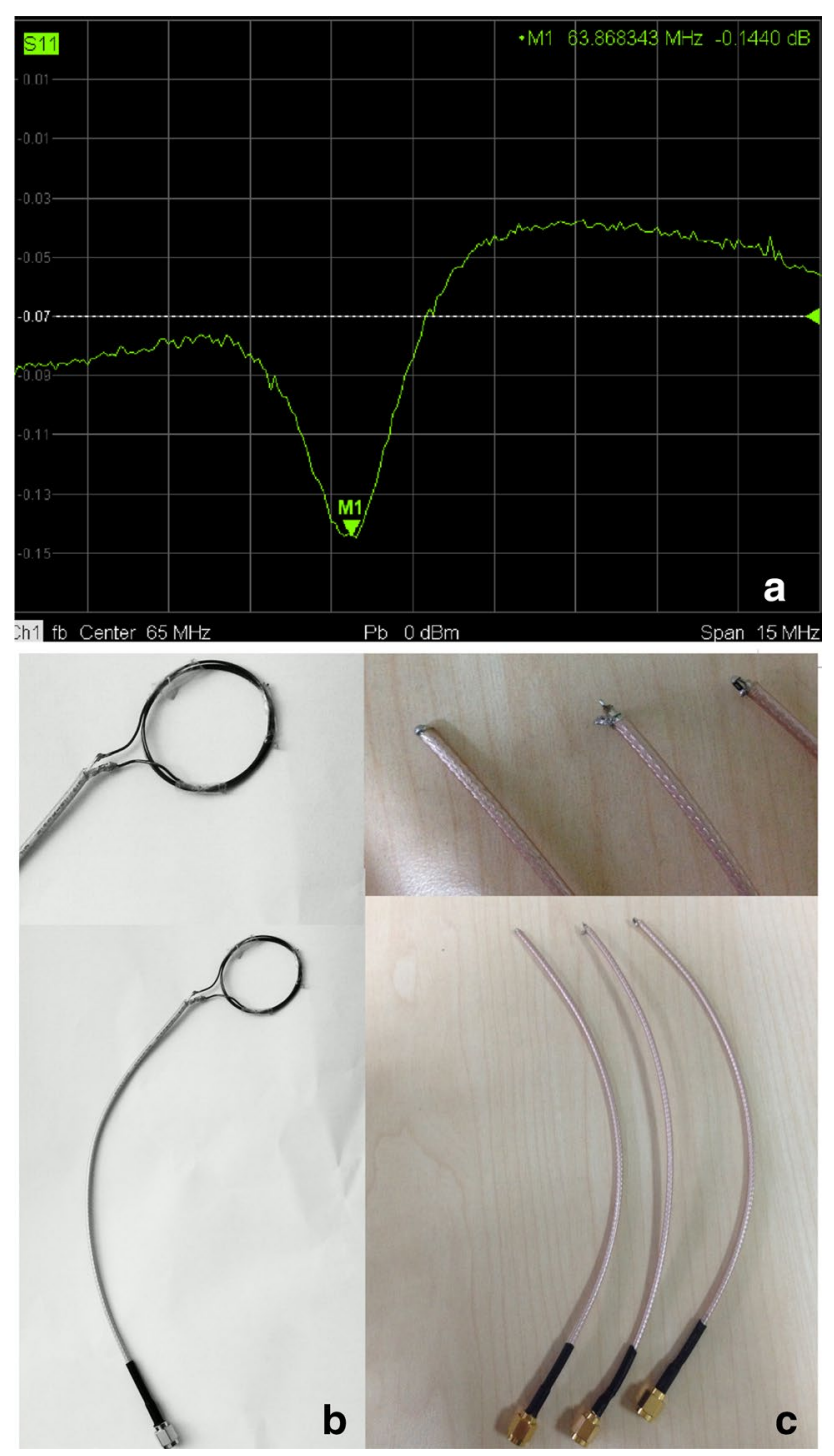

Fig. 6 Resonant frequency measurements of the RF resonant marker using a non-contact RF search probe. a Signal drop at the tuned frequency $(63.86 \mathrm{MHz})$ for the $\mathrm{S} 11$ measurement. b Photograph of the custom designed non-contact search probe and magnified view of the tip. c Photographs (left to right) of the calibration probes used for measurements (short circuit probe, open circuit probe and $50 \Omega$ matching probe) connected to the network analyzer and magnified views of their tips 

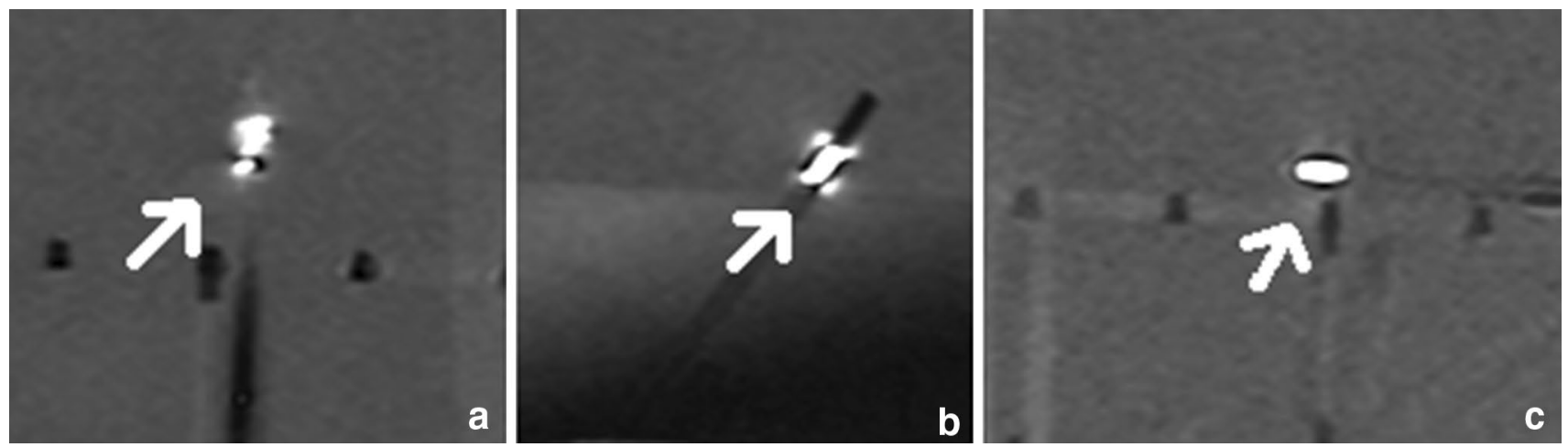

Fig. 7 The RF resonant marker appeared as a "bright spot" (indicated by white arrows) under MRI 1.5 T. Images show the MRI visibility of the RF resonant marker (Windowing parameters WL: 350,
WW: 650) when placed a parallel to the main magnetic field $B_{0}$, b oriented $45^{\circ}$ relative to $B_{0}$, and $\mathbf{c}$ placed perpendicular to $B_{0}$ 